American Journal of Infectious Diseases 3 (3): 134-141, 2007

ISSN 1553-6203

(C) 2007 Science Publications

\title{
Prevalence of Oncogenic HPV Types in Sardinia (Italy): Implications for the Impact of Prophylactic Anti-HPV Vaccines
}

\author{
${ }^{1}$ Stefania Montisci, ${ }^{1}$ Sabrina Pitzalis, ${ }^{1}$ Marianna Greco, ${ }^{2}$ Alberto Orani, ${ }^{3}$ Marco Rais and ${ }^{1}$ Sergio Laconi \\ ${ }^{1}$ Department of Biomedical Sciences and Biotechnology, University of Cagliari, Cagliari 09124, Italy. \\ ${ }^{2}$ Centro di Prevenzione Oncologica, ASL 8, Cagliari, Italy. \\ ${ }^{3}$ Unità di Anatomia Patologica, Ospedale Oncologico A. Businco, ASL 8, Cagliari, Italy
}

\begin{abstract}
Infection with high-risk HPV genotypes is considered an essential step in cervical carcinogenesis. Recently, prophylactic anti-HPV vaccines have shown to provide effective protection in clinical trials. However, protection appears to be type-restricted, thus, its real extent will depend on HPV types prevalence in the target population. Here we report HPV prevalence in 555 Italian women with various stages of HPV-related cervical disease (343 CIN1/L-SIL, 156 CIN2-3/HIGH-SIL, 43 invasive squamous cervical carcinoma and 13 adenocarcinoma), as well as in 315 women with smears negative for intraepithelial lesions or malignancy (NIL). HPV was found in $50.7 \%$ of CIN1/L-SIL, $87.2 \%$ of CIN2-3/HIGH-SIL, $97.7 \%$ of squamous carcinomas, $69.2 \%$ of adenocarcinomas and in $29.5 \%$ of NIL. HPV 16 was present in $69.0 \%$ of squamous carcinomas, $58.8 \%$ of CIN2-3/HIGH-SIL, in 22.9 and $16.1 \%$ of CIN1/L-SIL and NIL, respectively. HPV 18 was found in $7.3 \%$ and $4.8 \%$, respectively, while in $35.3 \%$ and $26.2 \%$ of CIN2-3/HIGH-SIL and invasive carcinomas, respectively, were present high-risk HPV types other than type 16 and 18 . Based on these data, it is expected that current HPV prophylactic vaccines could effectively prevent up to $70 \%$ of invasive cervical cancers and a slightly smaller proportion of high-grade lesions, in our population.
\end{abstract}

Keywords: HPV prevalence; HPV typing; High-grade lesions; Cervical cancer,

\section{INTRODUCTION}

It is well known that infection with high-risk Human Papilloma Viruses (HPVs) is a necessary step through the development of cervical cancer ${ }^{[1-3]}$. HPVs represent a large group of closely related, ubiquitary viruses that infect the skin and squamous epithelia throughout the body ${ }^{[4-6]}$. More than 100 types (genotypes) have been identified so far and new types, subtypes and variants continue to be added to the list. The so called anogenital HPVs infect preferentially the epithelial lining of the anogenital tract in both males and females, causing a wide range of proliferative lesions spanning from condilomata acuminata (genital warts) to cervical and anal cancer ${ }^{[5]}$. About 40 anogenital HPV types have been described, distinguished as either high-risk or low-risk, based on the frequency of their association with invasive cervical cancer ${ }^{[7-10]}$. Among the approximately 20 different high-risk HPV genotypes the most common worldwide are HPV 16, and HPV $18^{[7-10]}$.
HPV genotypes are identified according to the sequence of the L1 gene, which encodes for the major capsid protein of HPV. Type-specific neutralizing antibodies are produced against this protein during natural infection, but such a humoral response is thought to play little or no role in virus clearance ${ }^{[11,12]}$. On the other hand, it is commonly accepted that neutralizing antibodies against L1 offer protection against new infections by the same HPV type, but, being type restricted, they are considered to be largely ineffective against other HPV types. The humoral response is also thought to play no role in the regression of pre-invasive lesions and in advanced stages of cervical disease. For this reason, two approaches have been taken in the development of vaccine strategies against HPV: The first approach (prophylactic) is aimed to eliciting a powerful humoral response against the same antigen(s) (the L1 protein) that trigger(s) the antibody response to natural infection; the second approach (therapeutic) is aimed to the eradication of established HPV infections through the induction of a cell-mediated immune

Corresponding Author: $\quad$ Sergio Laconi, MD. Ph.D., Department of Biomedical Sciences and Biotechnology, Section of Experimental Pathology, University of Cagliari, Cagliari 09124, Italy. Phone: +39 0706758430 ; Fax: 39-070-662574 
response against the transforming viral proteins E6 and/or E7. While some success has been achieved in the regression of transplanted cervical carcinomas in laboratory animals [13], the development of E6/E7based, therapeutic HPV vaccines has not reached the clinical trial stage yet.

Conversely, L1-based prophylactic vaccines have proved to be highly effective in clinical trials ${ }^{[14]}$. One such vaccine, Merk's Gardasil, targets the two most prevalent high-risk HPV types (16 and 18), but also two low-risk types, HPV 6 and 11, the main viral types involved in the development of genital warts. Gardasil has recently obtained U.S. FDA approval for human use, while another vaccine developed by Smith-Kline Glaxo (Cervarix), targeted against HPV 16 and 18, is expected to follow soon. Both vaccines were able to induce high-titer, type-specific antibody responses (much higher than those obtained after natural infection) in clinical trials and they offered $100 \%$ protection against infection by either HPV 16 or $18^{[15-}$ 17]. It is expected that these vaccines will have a dramatic impact on the incidence of invasive cervical cancer as well as pre-invasive cervical lesions ${ }^{[14,18,19]}$. However, due to the long latency of cervical cancer, the decline in the incidence of this disease will not be apparent until 3 or 4 decades after the start of the immunization programs. Moreover, since, as compared to invasive cancer, a larger proportion of pre-invasive lesion may be caused by HPV types other than HPV 16 and $18^{[20-21]}$ it is predicted that the impact of vaccination on the incidence of these lesions will be less prominent.

The prevalence of the various HPV types varies among different populations and geographical areas ${ }^{[7,}$ ${ }^{10]}$. Although the reasons for such regional differences are not completely understood, due to the typerestriction of the immune response against HPV, it is evident that any accurate prediction on the efficacy of vaccines in at-risk populations has to be based on the local HPV type prevalence data. In this study we have assessed the prevalence of a panel of $25 \mathrm{HPV}$ genotypes on a population of Italian women with various stages of pre-invasive cervical lesions or invasive cancer.

\section{MATERIALS AND METHODS}

Sample collection: Eight hundred-fifty one cervical samples were collected between 2001 and 2004 at the Unit of Anatomic Pathology of the Oncology Hospital "A. Businco" and the Center for Tumor Prevention in
Cagliari, Italy from women with various cytological alterations, after informed written consent. Samples consisted of either exfoliated cervical cells or cervical biopsies. All cases were re-evaluated by an expert pathologist (M.R.) and classified according to Bethesda 2001 as follows: 325 cases displayed only inflammatory or non specific changes (reactive changes, metaplasia) with no signs of intraepithelial lesions or malignancy and were classified as NIL; 359 cases showed evident signs of HPV infection (koilocytosis) and/or mild atypia and were classified as either cervical intraepithelial neoplasia (CIN) grade 1, or low-grade squamous intraepithelial lesion (L-SIL); 167 cases showed signs of severe atypia or carcinoma in situ (CIS) and were classified as CIN grade 2-3 (CIN2-3), high-grade SIL (H-SIL) or CIS.

Table 1: Women enrolled in the study

\begin{tabular}{lcc}
\hline $\begin{array}{l}\text { Cytological/histological } \\
\text { diagnosis }\end{array}$ & Number & $\begin{array}{l}\text { Mean Age } \\
\text { (Range) }\end{array}$ \\
\hline NIL & 315 & $37.1(18-67)$ \\
CIN1/L-SIL & 343 & $34.8(18-66)$ \\
CIN2-3/CIS/H-SIL/ & 156 & $38.8(19-64)$ \\
SCC & 43 & $51.2(27-86)$ \\
AC & 13 & $58.4(39-76)$
\end{tabular}

Total 870

$\mathrm{NIL}=$ no intraepithelial neoplasia

$\mathrm{CIN}=$ cervical intraepithelial neoplasia

$\mathrm{SIL}=$ squamous intraepithelial lesion

$\mathrm{CIS}=$ carcinoma in situ; $\mathrm{SCC}=$ squamous cell carcinoma

$\mathrm{AC}=$ adenocarcinoma

In total, out of 922 samples, 52 did not yield any amplifiable DNA and were excluded from the study. The remaining 870 samples consisted of 315 NIL, 343 CIN1/L-SIL, 156 CIN2-3/H-SIL/CIS, 43 SCCs and 13 ACs. A list of samples used in the present study is reported in Table 1. Sixty-five samples of cell scrapings or biopsies from condilomata acuminata (genital warts) of external genitals or the perianal region, obtained after informed written consent from both female (19) and male (46) patients treated at the Division of Dermatology of the Oncology Hospital "A. Businco" in Cagliari, were also included in this study. 
Sample processing and PCR: Samples were digested with a buffer containing $50 \mathrm{mM}$ Tris- $\mathrm{HCl}, 1 \mathrm{mM}$ EDTA, $5 \%$ Tween 20 and $2 \mathrm{mg} / \mathrm{ml}$ proteinase $\mathrm{K}$, for 12 16 hours at $55^{\circ} \mathrm{C}$. An aliquot of crude lysate was used for the PCR. Quality of lysates was tested by PCR amplification of the beta globin gene with the following primers: forward 5'-CTTTCAGGGCAATAATGA-3', reverse 5'-TGGTAGCTGGATTGTAGC. Crude lysates that did not yield a beta globin product were extracted with phenol/chloroform and the extracted DNA reamplified with the same beta globin primers. Samples that remained negative for beta globin were considered not amplifiable and excluded from this study.

HPV genotyping was done by PCR-reverse hybridization as previously described ${ }^{[22]}$. Briefly, a segment of the L1 region was amplified with GP5+/GP6+ consensus primers ${ }^{[23]}$ and labeled during PCR through the incorporation of Dig-11-dUTP (Roche Applied Science, Mannheim, Germany). Labeled amplicons were then hybridized to a panel of 25 type specific probes (High-risk: HPV 16, 18, 26, 30, 31, 33, $35,45,51,52,56,58,59,68$ and 73; low-risk: HPV 6, $11,34,40,42,43,44,54$ and 70), previously immobilized to the surface of NucleoLink wells (NUNC, Denmark) and detected with a PODconjugated anti-Digoxigenin antibody (Roche Applied Science, Mannheim, Germany) and the tetra methyl benzidine (TMB) substrate (Sigma-Aldrich, Milan Italy).

\section{RESULTS}

Prevalence of HPV DNA in cervical samples: We first screened all samples with consensus primer-PCR as described in methods, in order to detect the presence of HPV L1 sequences. Overall, as reported in Table 2, among the 870 samples that yielded a product for the control gene, HPV DNA was found in $52.2 \%$. However, as expected, the prevalence of HPV varied greatly according to the severity of cellular atypia. HPV was found in $29.5 \%$ of NIL samples while more than $50 \%(50.7 \%)$ of low-grade lesions were found positive for HPV. This percentage increased to $87.2 \%$ in highgrade lesions and to $97.7 \%$ in SCC. By contrast less than $70 \%$ (69.2) of ACs resulted positive for HPV.

We then performed type-specific HPV genotyping by reverse hybridization to single DNA probes specific for
Table 2: Prevalence of HPV DNA in cervical samples HPV DNA

Positive Negative Total

\begin{tabular}{lccc}
\hline & & & \\
NIL & $93(29.5)$ & $222(70.5)$ & 315 \\
CIN1/L-SIL & $174(50.7)$ & $169(49.3)$ & 343 \\
CIN2-3/ & $136(87.2)$ & $20(12.8)$ & 156 \\
CIS/H-SIL/ & & & \\
SCC & $42(97.7)$ & $1(2.3)$ & 43 \\
AC & $9(69.2)$ & $4(30.8)$ & 13 \\
Total & $454(52.2)$ & $416(47.8)$ & 870 \\
\hline
\end{tabular}

Numbers between brackets represent percentage values.

$\mathrm{NIL}=$ no intraepithelial neoplasia;

$\mathrm{CIN}=$ cervical intraepithelial neoplasia;

$\mathrm{SIL}=$ squamous intraepithelial lesion.

$\mathrm{CIS}=$ carcinoma in situ;

$\mathrm{SCC}=$ squamous cell carcinoma;

$\mathrm{AC}=$ adenocarcinoma.

HPV vaccines, namely HPV 16 and 18, a cocktail of probes for other high risk HPVs and a cocktail of probes for low-risk types. (See Methods for the complete list). As shown in Table 3, we found that HPV 16 was the most prevalent type in invasive cancers as well as in high-grade lesions, accounting for $69.0 \%$ and 58.8 of positive cases, respectively. By contrast, HPV 18 was present in only $4.8 \%$ of SCC and $7.3 \%$ of high-grade lesions, although, in line with data in the literature, the prevalence of this type was somewhat higher in AC. Thus, these two genotypes, alone or in mixed-type infections, were present in $73.8 \%$ of SCC cases and in $66.1 \%$ of high-grade lesions. On the other hand, $26.2 \%$ of SCC and $35.3 \%$ of high-grade lesion were positives for other high-risk HPV types, either alone or in combination with HPV 16 or 18.

As expected, HPV 16 was present in a smaller percentage of low-grade lesions and NIL cases (22.9 and $16.1 \%$, respectively), while, surprisingly, HPV 18 was more frequently found in these (12.6 and $15.0 \%$ of

CIN1/L-SIL and NIL, respectively), as opposed to high-grade lesions and SCC.

Importantly, HPV types other than 16 and 18 were present in about $2 / 3$ of low-grade lesions. 
Am. J. Infect. Dis., 3 (3): 134-141, 2007

Table 3: Prevalence of HPV 16 and 18 among HPV positive cervical samples

\begin{tabular}{|c|c|c|c|c|c|c|c|}
\hline & HPV $16^{a}$ & $\underset{\mathrm{a}}{\mathrm{HPV}} 18$ & Other $\mathrm{HR}^{\mathrm{a}}$ & $\begin{array}{c}\text { Mixed } \\
\text { HR }\end{array}$ & $\begin{array}{l}\text { Mixed } \\
\text { HR/LR }\end{array}$ & LR & ND \\
\hline $\begin{array}{l}\text { NIL } \\
\mathrm{N}=93\end{array}$ & $\begin{array}{c}15 \\
(16.1)\end{array}$ & $\begin{array}{c}14 \\
(15.0)\end{array}$ & $\begin{array}{c}56 \\
(60.2)\end{array}$ & $\begin{array}{c}13 \\
(13.9)\end{array}$ & $\begin{array}{c}16 \\
(17.2)\end{array}$ & $\begin{array}{c}33 \\
(35.5)\end{array}$ & $\begin{array}{c}4 \\
(4.3)\end{array}$ \\
\hline $\begin{array}{l}\text { CIN1/L-SIL } \\
\mathrm{N}=174\end{array}$ & $\begin{array}{c}40 \\
(22.9 \%)\end{array}$ & $\begin{array}{c}22 \\
(12.6)\end{array}$ & $\begin{array}{c}81 \\
(46.5 \%\end{array}$ & $\begin{array}{c}23 \\
(13.2)\end{array}$ & $\begin{array}{c}24 \\
(13.8)\end{array}$ & $\begin{array}{c}37 \\
(21.2)\end{array}$ & $\begin{array}{c}10 \\
(5.7)\end{array}$ \\
\hline $\begin{array}{l}\text { CIN2-3/CIS/H-SIL/ } \\
\mathrm{N}=136\end{array}$ & $\begin{array}{c}80 \\
(58.8)\end{array}$ & $\begin{array}{c}10 \\
(7.3)\end{array}$ & $\begin{array}{c}48 \\
(35.3)\end{array}$ & $\begin{array}{c}9 \\
(6.6)\end{array}$ & $\begin{array}{c}1 \\
(0.7)\end{array}$ & $\begin{array}{c}5 \\
(3.6)\end{array}$ & $\begin{array}{c}4 \\
(2.9)\end{array}$ \\
\hline $\begin{array}{l}\mathrm{SCC} \\
\mathrm{N}=42\end{array}$ & $\begin{array}{c}29 \\
(69.0)\end{array}$ & $\begin{array}{c}2 \\
(4.8)\end{array}$ & $\begin{array}{c}11 \\
(26.2)\end{array}$ & $\begin{array}{c}1 \\
(2.4)\end{array}$ & -- & -- & $\begin{array}{c}1 \\
(2.4)\end{array}$ \\
\hline $\begin{array}{l}\mathrm{AC} \\
\mathrm{N}=9\end{array}$ & $\begin{array}{c}4 \\
(44.4) \\
\end{array}$ & $\begin{array}{c}2 \\
(22.2) \\
\end{array}$ & $\begin{array}{c}3 \\
(33.3) \\
\end{array}$ & -- & -- & -- & -- \\
\hline
\end{tabular}

Table 4: Prevalence of 25 anogenital HPV in cervical samples

\begin{tabular}{|c|c|c|c|c|}
\hline HPV type & CIN1/L-SIL & CIN2-3/CIS/H-SIL/ & SCC & $\mathrm{AC}$ \\
\hline HPV 6 & $8(12.7)$ & - & - & - \\
\hline HPV 16 & $16(25.4)$ & $41(56.9)$ & $29(69.0)$ & $4(44.4)$ \\
\hline HPV 18 & $7(11.1)$ & $5(6.9)$ & $2(4.8)$ & $2(22.2)$ \\
\hline HPV 30 & $1(1.6)$ & - & - & - \\
\hline HPV 31 & $9(14.3)$ & $4(5.5)$ & $1(2.4)$ & - \\
\hline HPV 33 & $1(1.6)$ & $9(12.5)$ & $3(7.1)$ & - \\
\hline HPV 35 & $3(4.7)$ & $2(2.8)$ & $1(2.4)$ & - \\
\hline HPV 40 & $1(1.6)$ & - & - & - \\
\hline HPV 42 & $15(23.8)$ & $1(1.4)$ & - & - \\
\hline HPV 43 & $1(1.6)$ & - & - & - \\
\hline HPV 44 & $2(3.2)$ & - & - & - \\
\hline HPV 45 & $1(1.6)$ & $2(2.8)$ & $2(4.8)$ & $1(11.1)$ \\
\hline HPV 51 & $4(6.3)$ & $6(8.3)$ & $1(2.4)$ & $1(11.1)$ \\
\hline HPV 52 & $1(1.6)$ & $1(1.4)$ & - & - \\
\hline HPV 56 & $6(9.5)$ & - & $1(2.4)$ & - \\
\hline HPV 58 & $2(3.2)$ & $2(2.8)$ & - & - \\
\hline HPV 59 & $1(1.6)$ & - & - & - \\
\hline HPV 68 & - & $1(1.4)$ & - & - \\
\hline HPV 70 & $1(1.6)$ & - & - & - \\
\hline HPV 73 & $1(1.6)$ & $1(1.4)$ & $2(4.8)$ & - \\
\hline ND & $3(4.7)$ & $2(2.8)$ & $1(2.4)$ & - \\
\hline Total & 63 & 72 & 42 & 9 \\
\hline
\end{tabular}

In order to investigate the real prevalence of specific HPV types, in addition to HPV 16 and 18, we fully genotyped a randomly selected group of lowgrade (63) and high-grade (72) lesions, as well as all cases of invasive cancer. As shown in table 4, HPV 16 was still the most prevalent type in this selected group of low-grade lesions (25.4\%), however the low-risk type HPV 42 was almost equally represented. Importantly, as evident from tables 3 and 4, when compared to other high-risk types, HPV 16 prevalence increases sharply according to the severity of the atypia, going from about $16 \%$ in the NIL group, to almost $70 \%$ 
in SCC cases. This data are in line with the findings of others (20) and suggest that infections sustained by HPV 16 are more prone to persist and cause progressive lesions as compared to other types. Conversely, the prevalence of HPV 18 showed an opposite trend in squamous lesions, being more prevalent in low as opposed to high-grade lesions and SCC.

In fact, in the present study, HPV 18 was found in less that $5 \%$ of cases of invasive SCC, similarly to both HPV 45 and 73. In our study, the most prevalent type in both SSC and high-grade lesions, after HPV 16, was the closely related type HPV $33 \quad(7.1 \%$ and $12.5 \%$, respectively), while HPV 31, 35, 51 and 56 were each found in only one case $(1 / 42,2.4 \%)$. As shown in table 4, overall, the spectrum of high-risk HPV types was much broader in low-grade lesions and NIL, as compared to High-grade lesions and invasive cancer. As a final point, since the only HPV vaccine currently approved by FDA in addition to HPV 16 and 18, also targets the low-risk types HPV 6 and 11, the genotypes most notoriously associated to the development of condilomata acuminata (genital warts) ${ }^{[24,25]}$, we wanted to assess the prevalence of these HPV types in tissue biopsies and cell scrapings from lesions of external genitalia and the perianal region from both male and female patients. As shown in Table 5, and in keeping with established data, $80 \%(52 / 65)$ of genital warts analyzed in our study contained the DNA of either HPV 6 or 11, while high risk genotypes or other low-risk types were found in a minority of cases, either alone or associated with HPV 6/11. These data indicate that immunization with an HPV vaccine targeted to genotypes 6 and 11, in addition to 16 and 18, besides representing a valuable tool in the prevention of a great proportion of cervical cancers could be extremely effective in the prophylaxis of genital warts in our population.

Table 5: Prevalence of HPV genotypes in genital warts of external genitalia and the perianal region.

\begin{tabular}{lc}
\hline HPV Type & HPV Prevalence \\
\hline HPV 16 & $4(7.0)$ \\
HPV 18 & - \\
Other HR & $6(9.2)$. \\
HPV 6/11 & $52(80.0)$ \\
Other LR & $6(9.2)$ \\
Mixed & $8(12.3)$ \\
Negative & $6(9.2)$ \\
Total & 65 \\
\hline HR = High-Risk HPV types; LR = Low-Risk HPV types; Numbers \\
between brackets represent percentage values.
\end{tabular}

\section{DISCUSSION}

Within the last 2 decades, progress in the understanding of cervical cancer biology has had a profound effect on how we approach this disease. The notion that infection with high-risk HPV types is a necessary, though not sufficient, step in the development of cervical as well other cancers of the anogenital tract has already forced us to reconsider screening strategies for these diseases. More recently, the arrival on the market of anti-HPV prophylactic vaccines promises to revolutionize current preventive strategies for HPV-related cancers. Unfortunately, not unlike other highly adapted human viruses, HPV exists in nature in a great number of serotypes and variants that elicit largely non-cross-reactive, type-restricted immune responses ${ }^{[12]}$. Thus one of the major challenges to the success of HPV vaccines would be their range of type-effectiveness.

Current HPV prophylactic vaccines were designed so as to target only two high-risk HPV types, with the notion that these represent the most prevalent types in cervical cancer cases worldwide [7, 10]. However differences in the prevalence among different populations are well known and are likely to have an impact on vaccine efficacy. Thus, actual prevalence data in different populations can be very valuable in monitoring vaccine efficacy during clinical studies and they could also be instrumental in designing more effective, population-tailored vaccine formulations that incorporate the most prevailing types in any given geographical area.

The main goal of our work was to gather information on the prevalence of HPV 16 and 18, the two high risk HPV types included in the prophylactic vaccines under clinical trial, in those cervical lesions that are currently considered for ablative treatment, namely CIN2/HSIL or higher, in order to be able to predict the possible clinical benefits brought by the implementation of the HPV vaccine in our region. To the best of our knowledge this is the largest such study ever reported on Italian women, including over 200 cases of high-grade cervical lesions and invasive carcinomas. In keeping with published reports in Italian women and worldwide ${ }^{[20,26]}$, the prevalence of HPV infection in our study varied according to the degree of cervical atypia, approaching $100 \%$ in squamous cell carcinoma, although, as reported in most studies ${ }^{[10]}$, prevalence remained significantly lower in ACs.

Almost $30 \%$ of women with NIL smears were positive for HPV, a value somewhat higher than 
expected for the general population of this age group (mean age 37.1 years). However, though negative for intraepithelial lesion or malignancy, the women of this group underwent reflex HPV DNA testing because of non-specific alterations in their smears. As such they represent a selected group of women that cannot be considered representative of the general population.

In line with known epidemiological data from Italy ${ }^{[26-}$ ${ }^{28]}$ and other western Countries ${ }^{[7,10]}$, we found that HPV 16 is by far the most prevalent type in SCC and high-grade lesions, accounting for almost $70 \%$ of positives cases among SCCs in our study. Conversely, in contrast to most epidemiological surveys ${ }^{[7,10]}$, our data would suggest that HPV 18 does not play a major role in the development SCC in our population. In fact, in our study the prevalence of this HPV type was much higher in low-grade lesions as compared to high-grade squamous lesions and invasive carcinomas. It is obvious that the natural history of HPV 18 infection in our population cannot be inferred solely by single point prevalence data, however our findings would suggest that, in contrast to what reported by others [29,30], infection with this HPV type is not associated with highly progressive lesions in our population. It is obvious that the prevalence of some high-risk HPV types might be underestimated in high-grade lesions, as compared to low-grade lesions and NIL, due to the small number of cases examined. Nonetheless, their relative rarity in these lesions may reflect their scarce tendency to cause persistent infections and progressive pre-invasive lesions.

In summary, our data indicate that almost $75 \%$ of SCC and $65 \%$ of high-grade lesions in our region are due to infection with either HPV 16 or 18. Assuming that immunization programs will achieve $100 \%$ protection rates against infection with either HPV 16 or 18 in vaccinated women (as reported in phase III clinical trials), at least in theory we could expect to prevent up to $75 \%$ of cervical cancers solely by vaccination, in our region. Obviously, these theoretical considerations will have to be weighted against the degree of vaccine coverage actually reached in the population at risk. The true challenge for the near future will be to obtain the proper extent of vaccine coverage in those women who are also less likely to adhere to screening programs and as such are at greater risk of cervical cancer.

After HPV 16, the viral type most frequently found in SCCs in our study was HPV 33, a type not included in current vaccine formulations, but closely related to HPV 16. Interestingly, recent reports on phase III clinical trials ${ }^{[17]}$ would suggest that the bivalent GSK vaccine might be able to offer limited but significant protection against HPV types closely related to either
HPV 16 (such as HPV 31) or HPV 18 (such as HPV 45). While further studies are needed to confirm these reports, our data indicate that about $1 / 4$ of SCC and $1 / 3$ of high-grade lesions harbor HPV types not included in current vaccine formulation, thus, even in vaccinated populations, screening for cytological alteration or/and HPV DNA will continue to be a very important tool in the fight against cervical cancer. In fact, recently issued American Cancer Society guidelines ${ }^{[31]}$ recommend that "women undergo regular screening regardless of whether they have been vaccinated".

Screening programs based on Pap smears and HPV DNA tests will continue to be the main preventive tool for women 19 or older, in which vaccination is not recommended ${ }^{[31]}$. In this contest, it is important to note that our data show a clear trend towards increasing prevalence values of HPV 16 infection with increasing grades of atypia. We take this as indirect evidence that HPV 16 is much more prone to cause persistent infections and progressive lesions, as compared to other high-risk types, a conclusion well supported by more direct evidence from prospective studies ${ }^{[32-34]}$. Since viral persistence is considered necessary prerequisite for the development of progressive lesions ${ }^{[35]}$ and since it is estimated that up to $30 \%$ of women infected with persistent HPV 16 will develop high-grade lesions or cancer within 10 years ${ }^{[32,33]}$, we, as others ${ }^{[34]}$, suggest that the women persistently infected with HPV 16 may deserve a closer follow-up as compared to those infected with other high-risk types, even in the absence of any Pap smear alteration.

Genital warts, although entirely benign, represent a major factor of morbidity and among the most common sexually transmitted diseases. As confirmed in this paper, the vast majority of these lesions are caused by infection with HPV 6 or $11^{[24,25]}$. In this respect, the association of these two HPV types with HPV 16 and 18 in a polyvalent vaccine formulation may prove to be a clever strategy for the prevention of this disease in addition to cervical cancer.

In conclusion, although many important issues, such as cost/benefit analysis, vaccine delivery and acceptance by the target population (and the communities), still await to be fully investigated, from a clinical standpoint, the introduction of HPV vaccines is likely to offer a very effective tool for the prevention of cervical as well as other HPV related neoplasias. Whether this will translate in net gains for the women and the health care system in our geographical area, will depend largely on the choice of the right vaccine implementation strategies. 


\section{REFERENCES}

1. zur Hausen, H., E.M. de Villiers and L. Gissmann, 1981. Papillomavirus infections and human genital cancer. Gynecol. Oncol., 2: S124-S128.

2. Walboomers, J.M.M., M.V. Jacobs, M.M. Manos et al, 1999. Human papillomavirus is a necessary cause of invasive cervical cancer world-wide. J. Pathol., 189: 12-19.

3. Schiffman, M.H., H.M. Bauer, R.N. Hoover et al., 1993. Epidemiologic evidence showing that human papillomavirus infection causes most cervical intraepithelial neoplasia. J. Natl. Cancer. Inst., 85: 958-64.

4. Koutsky, L.A., D.A. Galloway and K.K. Holmes, 1988. Epidemiology of genital human papillomavirus infection. Epidemiol. Rev., 10: 12263.

5. Alani, R.M. and K. Munger, 1998. Human papillomaviruses and associated malignancies. J. Clin. Oncol., 16: 330-337.

6. zur Hausen, H., 1996. Papillomavirus infection - a major cause of human cancers. Biochem. Biophys. Acta, 1288: F55-F78.

7. Bosch, F.X., M.M. Manos, N. Munoz et al., 1995. International Biological Study on Cervical Cancer (IBSCC) Study Group. Prevalence of human papillomavirus in cervical cancer: a worldwide perspective. J. Natl. Cancer Inst., 87: 796-802.

8. Lorincz, A.T., R. Reid, A.B. Jenson, M.D. Greenberg, W. Lancaster and R.J. Kurman, 1992. Human papillomavirus infection of the cervix: relative risk associations of 15 common anogenital types. Obstet. Gynecol., 79: 328-337.

9. Muñoz, N., F.X. Bosch, S. de Sanjosé et al., 2003. For the International Agency for Research on Cancer Multicenter Cervical Cancer Study Group. Epidemiologic classification of human papillomavirus types associated with cervical cancer. N. Engl. J. Med., 348: 518-527.

10. Clifford, GM, J.S. Smith, M. Plummer, N. Munoz and S Franceschi, 2003. Human papillomavirus types in invasive cervical cancer worldwide: a meta-analysis. Br. J. Cancer, 88 :63-73.

11. Frazer, I., 1998. The role of the immune system in Human papillomavirus infection. J. Obstet. Gyn., 18: S72-S75.

12. Stanley, M., 2006. Immune responses to human papillomavirus. Vaccine, 24 (S1 1): S16-22.

13. Zwaveling, S., S.C.F. Mota, J. Nouta et al., 2002. Established human papillomavirus type 16expressing tumors are effectively eradicated following vaccination with long peptides. J. Immunol., 169: 350-358.
14. Lowy, D.R. and J.T. Shiller, 2006. Prophylactic human papillomavirus vaccines. J. Clin. Invest., 116: 1167:1173.

15. Villa, L.L., R.L. Costa, C.A. Petta et al., 2006. High sustained efficacy of a prophylactic quadrivalent human papillomavirus types 6/11/16/18 L1 virus-like particle vaccine through 5 years of follow-up. Br. J. Cancer, 95:1459-1466.

16. Harper, D.M., E.L. Franco, C. Wheeler et al., 2004. GlaxoSmithKline HPV Vaccine Study Group. Efficacy of a bivalent L1 virus-like particle vaccine in prevention of infection with human papillomavirus types 16 and 18 in young women: a randomised controlled trial. Lancet, 364: 17571765.

17. Harper, D., E. Franco, C. Wheeler et al., 2006. HPV vaccine Study group. Sustained efficacy up to 4.5 years of a bivalent L1 virus-like particle vaccine against human papillomavirus types 16 and 18: follow-up from a randomised control trial. Lancet, 367: 1247-1255.

18. Hymel, P.A., 2006. Decreasing the risk: Impact of HPV vaccination on outcomes. Am. J. Manag. Care, 12: S473-S483.

19. Steinbrook, R., 2006. The Potential of Human Papillomavirus Vaccines. N. Engl. J. Med., 354: 1109-1112.

20. Clifford, G.M., R.K. Rana, S. Franceschi, J.S. Smith, G. Gough and J.M. Pimenta, 2005. Human papillomavirus genotype distribution in low-grade cervical lesions: comparison by geographic region and with cervical cancer. Cancer Epidemiol. Biomarkers Prev., 14: 1157-1164.

21. Clifford, G.M., J.S. Smith, T. Aguado and S. Franceschi, 2003. Comparison of HPV type distribution in high-grade cervical lesions and cervical cancer: a meta-analysis. Br. J. Cancer, 89: 101-105.

22. Laconi, S., M. Greco, P. Pellegrini-Bettoli, M. Rais, E. Laconi and P Pani, 2001. One-step detection and genotyping of human papillomavirus in cervical samples by reverse-hybridization. Diagn. Mol. Pathol., 10 (3): 200-206.

23. de Roda Husman, A.M., J.M.M. Walboomers, A.J.C. Van Den Brule, C.J.L.M. Meijer and P.J.F. Snijders, 1995. The use of general primers GP5 and GP6 elongated at their $3^{\prime}$ ends with adjacent highly conserved sequences improves human papillomavirus detection by PCR. J. Gen. Virol., 76: 1057-1062.

24. Tomita, Y., K. Kubota, T. Kasai, S. Sekiya, H. Takamizawa and B Simizu, 1986. Detection of human papillomavirus DNA in genital warts, cervical dysplasias and neoplasias. Intervirology, 25: $151-157$. 
25. Felix, J.F. and T.C. Wright, 1994. Analysis of lower genital tract lesions clinically suspicious for condylomata using in situ hybridization and the polymerase chain reaction for the detection of human papillomavirus. Arch. Pathol. Lab. Med., 118: 39-43.

26. Tornesello, M.L., M.L. Duraturo, G. Botti et al., 2006. The Italian HPV working group. Prevalence of alpha-papillomavirus genotypes in cervical squamous intraepithelial lesions and invasive cervical carcinoma in the italian population. J. Med .Virol., 78: 1663-1672.

27. Gargiulo, F., De M.A. Francesco, C. Schreiber et al., 2007. Prevalence and distribution of single and multiple HPV infections in cytologically abnormal cervical samples from Italian women. Virus Res., in press.

28. Del Mistro, A., H.F. Salamanca, R. Travisan et al., 2007. Human papillomavirus typing of invasive cervical cancer. Infect. Agent Cancer, 1: 9.

29. Kurman, R.J., M.H. Schiffman, W.D. Lancaster et al., 1988. Analysis of individual human papillomavirus types in cervical neoplasia: a possible role for HPV 18 in rapid progression. Am. J. Obstet. Gynecol., 159: 293-296.

30. Arends, M.J., Y.K. Donaldson, E. Duvall, A.H. Wyllie and C.C. Bird, 1993. Human papillomavirus type 18 associates with more advanced cervical neoplasia than human papillomavirus type 16. Human. Pathol., 24: 432437.
31. Saslow, D., P.E. Castle, J.T. Cox et al., 2007. American Cancer Society Guideline for Human Papillomavirus (HPV) Vaccine Use to Prevent Cervical Cancer and Its Precursors. CA Cancer J. Clin., 57: 7-28.

32. Khan, M.J., P.E. Castle, A.T. Lorincz et al., 2005. The elevated 10-year risk of cervical precancer in women with human papillomavirus (HPV) type 16 or 18 and the possible utility of type-specific HPV testing in clinical practice. J. Natl. Cancer Inst., 97: 1072-1079.

33. Castle, P.E., D. Solomon, M. Shiffman and C.M. Wheeler, 2005. Human papillomavirus type 16 infections and 2-year absolute risk of cervical precancer in women with equivocal or mild cytologic abnormalities. J. Natl. Cancer Inst., 97: 1066-1071.

34. Shiffman, M., R. Herrero, R. De Salle et al., 2005. The carcinogenicity of human papillomavirus types reflects viral evolution. Virology, 332: 76-84.

35. Ho, G.Y.F., R.D. Burk, S. Klein et al., 1995. Persistent genital human papillomavirus infection as a risk factor for persistent cervical dysplasia. J. Natl. Cancer Inst., 87: 1365-1371. 